\title{
Drought Stress Response of Indian Mustard (Brassica juncea L.) Genotypes
}

\author{
V.V. Singh, Pankaj Garg ${ }^{*}$, H.S. Meena and M.L. Meena \\ ICAR-Directorate of Rapeseed Mustard Research, Sewar Bharatpur, India \\ *Corresponding author
}

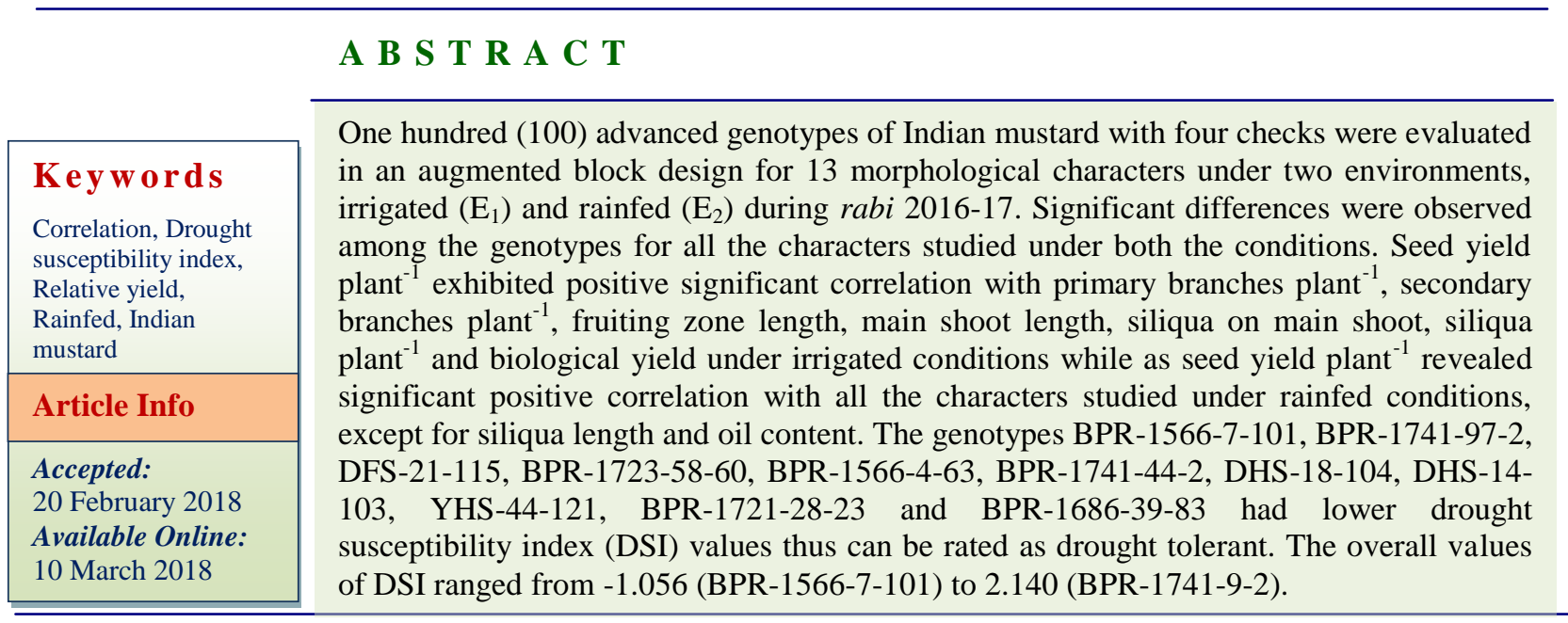

\section{Introduction}

Rapeseed mustard group of crops are grown under diverse agro-climatic conditions in India ranging from north-eastern or north western hills to down south under-irrigated or rain-fed area either timely or late sown in saline soils and mixed cropping systems. They are mainly cultivated in the northern and eastern parts of the country. Generally, Brassica species have been developed in the areas with high rainfall so perform poorly in the areas with low rainfall (Resketo and Szabo, 1992; Richards, 1978). Growth and seed yield production of Brassica species have greatly decreased owing to drought conditions. Like other crop, mustard suffers from stringent droughts with fluctuating and un-predicting intensities, so there is an urgent need for drought proofing of mustard crop. Fischer and Maurer (1978) gave drought susceptibility index (DSI) which characterizes the stability of yield between two environments. The most widely used criteria for high yielding genotypes are mean seed yield, mean productivity, and relative yield performance under moisture stress and normal environments. The relative seed yield has also been proposed as a useful selection criterion for assessing drought response of a genotype (Ahmed et al., 1999). The knowledge about the magnitude of genetic parameters of seed yield and its component traits for different conditions is essential for an effective breeding programme. Moreover, 
association and interaction of different component characters with seed yield may help in the selection of superior lines raised under varied environments. Yield a very complex trait, possesses many components characters which finally result in a highly plastic yield structure (Meena et al., 2014). The inter-relationships between characters help the plant breeder to assess the nature, extent and direction of selection on characters. Keeping this in view, the present investigation was undertaken to identify drought tolerant genotypes which may be further advanced to evaluate under multi-location trials for identification and development of drought tolerant cultivars of Indian mustard.

\section{Materials and Methods}

The experimental material for the study consisted of 100 advanced progenies of Indian mustard (Brassica juncea L.) developed under systematic breeding programme. The experiments were carried out at ICARDirectorate of Rapeseed-Mustard Research, Bharatpur (India) during 2016-2017 in an augmented block design (Fedrer, 1956) under normal $\left(\mathrm{E}_{1}\right)$ and water-stressed $\left(\mathrm{E}_{2}\right)$ conditions. The genotypes were raised in two rows of $5 \mathrm{~m}$ length with a spacing of $30 \mathrm{~cm}$ between rows and $10 \mathrm{~cm}$ between plants. The proper spacing between plants was achieved through thinning after 15-20 days of sowing. The genotypes evaluated were divided into five blocks consisting of 20 progenies and four check varieties in each block, namely, DRMRIJ-31, NRCDR-02, Kranti and RH-749. Border effect was removed by taking observations on middle plants in a row. Waterstressed conditions were maintained for rainfed experiment $\left(E_{2}\right)$ where only pre-sowing irrigation was applied, while as in normal irrigated $\left(\mathrm{E}_{1}\right)$ environment the treatments were irrigated twice, 35 DAS and 70 DAS. Initial moisture content of the experimental field at sowing was $5.07 \%(0-15 \mathrm{~cm}$ depth $), 6.76 \%$
$(15-30 \mathrm{~cm})$ and $12.07 \%(30-45 \mathrm{~cm})$. After 60 days, moisture content under rainfed conditions was recorded which was reduced to $2.53,2.81$ and $6.01 \%$ at respective soil depth. Observations for 13 morphological characters viz., plant height, numbers of primary branches plant $^{-1}$, numbers of secondary branches plant ${ }^{-1}$, fruiting zone length, main shoot length, numbers of siliquae on main shoot, numbers of siliquae plant $^{-1}$, siliqua length, numbers of seeds siliqua ${ }^{-1}$, biological yield plant $^{-1}$, seed yield plant ${ }^{-1}, 1000$-seed weight and oil content were taken. The mean data were subjected to analysis of variance (Fedrer, 1956) and analysis done through computer generated programme SPAD (Abhishek et al., 2004) software. Genetic parameters and simple correlations in all possible combinations were worked out as per standard procedure (Burton, 1952; Johnson et al., 1955). The relative drought tolerance of genotypes was quantified with respect to seed yield through DSI according to Fischer and Maurer (1978). The relative yield under rainfed/normal (irrigated) was calculated as the yield of a specific genotype under rainfed/ irrigated divided by that of the yield of highest yielding genotype in the population.

\section{Results and Discussion}

Overall mean performance of progenies was comparatively higher in irrigated environment as compared to rain-fed condition (Table 1).

Under water stress conditions the decreased translocation of assimilates/growth substances and loss of turgidity might be the reason for lower mean performance of progenies as described by Kumawat et al., (1997). Singh and Choudhary (2003) and Chauhan et al., (2007) reported up to $60 \%$ yield reductions under rainfed condition in Brassica juncea. The magnitude of phenotypic coefficient of variation (PCV) was higher than the corresponding genotypic coefficient of 
variation (GCV) for all the characters under both the environmental conditions suggesting the role of environment in expression of these characters. It was observed that the number of siliquae plant ${ }^{-1}$ followed by biological yield and number of siliqua on main shoot under irrigated condition and number of siliquae plant $^{-1}$ followed by biological yield and length of fruiting zone under rain-fed condition exhibited comparatively higher estimates of both GCV and PCV (Table 1). It indicated that simple selection for these characters might be advantageous in particular condition. Similar findings were earlier reported by Singh et al., (2007).

The estimates of broad sense heritability were higher in magnitude $(>50 \%)$ for all the characters under both the conditions with significant variability except for siliqua length under irrigated condition. These findings are in accordance with the results reported by Singh et al., (2009) and Meena et al., (2008).

The genetic advance as percent of mean was highest for siliqua plant ${ }^{-1}$ followed by biological yield and plant height under both irrigated and rainfed conditions indicating that selection for these traits would be effective for the improvement and there is better scope for development of genotypes having more number of siliqua plant ${ }^{-1}$, biological yield, increased fruit zone length, higher number of siliqua on main shoot.

Similarly, there is enough scope for development of promising genotypes for water stress conditions having increased siliqua plant $^{-1}$, biological yield and more number of siliqua on main shoot. High heritability with high genetic advance for siliqua plant ${ }^{-1}$, biological yield, plant height and siliqua on main shoot under irrigated conditions while siliqua plant $^{-1}$, biological yield, plant height and fruiting zone length under rainfed are indicative of additive gene action for these traits. High heritability with high genetic advance for these characters has also been reported (Patel et al., 2006; Singh et al., 2011 and Tiwari et al., 2017) which supports the results of the present investigation. High heritability with low genetic advance were observed for siliqua on main shoot, test weight, secondary branches plant ${ }^{-1}$ and seeds siliqua under irrigated conditions while under rainfed condition seed yield, oil content, test weight and seeds siliqua ${ }^{-1}$. It indicated more influence of non-additive gene effects so selection in later generation would be more effective for these traits.

Correlation coefficient analysis measures relation between various plant characters and determines the component characters on which selection can be used for genetic improvement in yield. The lower values of correlation may be attributed to lower modifying effect of environment on the association of characters at gene level (Mamun-Hossain and Joarder, 1987; Ramya et al., 2012). Under irrigated conditions (Table 2) seed yield plant $^{-1}$ was positively and significantly correlated with primary branches per plant (0.393), secondary branches plant ${ }^{-1}$ (0.386), fruiting zone length (0.168), main shoot length (0.131), siliqua on main shoot (0.187), siliqua plant ${ }^{-1}(0.436)$, biological yield (0.600)while seed yield plant $^{-1}$ was significantly and positively correlated with plant height $(0.432)$, primary branches plant $^{-1}$ (0.433), secondary branches plant ${ }^{-1}(0.406)$, fruiting zone length (0.405), main shoot length (0.426), siliqua on main shoot (0.430), siliqua plant $^{-1} \quad(0.554)$, seeds siliqua ${ }^{-1} \quad(0.201)$, biological yield (0.712) and test weight (0.353) under rainfed conditions. These findings are in agreement with the earlier reports of Kardam and Singh (2005), Meena et al., (2008), Roy et al., (2016), Doddabhimappa et al., (2009), Singh et al., (2009, 2011 \& 2015), Dipti (2016) and Meena et al., (2017). 
Table.1 Estimates of mean, range, GCV, PCV, heritability (bs) and genetic advance for seed yield and its components in advanced progenies of Indian mustard under

Rainfed and irrigated conditions

\begin{tabular}{|c|c|c|c|c|c|c|c|c|}
\hline Trait & Condition & Mean & $\begin{array}{c}\% \text { reduction in } \\
\text { mean }\end{array}$ & Range & GCV & PCV & Heritability (broad sense) & Genetic advance as \% of mean \\
\hline \multirow[t]{2}{*}{ PH } & IR & 191.28 & \multirow[t]{2}{*}{9.56} & $155.00-230.00$ & 7.63 & 9.51 & 64.34 & 17.44 \\
\hline & RF & 173.00 & & $144.20-256.60$ & 10.55 & 11.34 & 86.59 & 26.60 \\
\hline \multirow[t]{2}{*}{ PB } & IR & 6.09 & \multirow[t]{2}{*}{6.57} & $4.20-9.00$ & 2.58 & 3.33 & 60.15 & 1.02 \\
\hline & $\mathbf{R F}$ & 5.69 & & $4.20-7.60$ & 2.57 & 3.09 & 68.99 & 1.05 \\
\hline \multirow[t]{2}{*}{ SB } & IR & 11.45 & \multirow[t]{2}{*}{21.05} & $7.60-22.50$ & 6.63 & 7.31 & 82.32 & 4.19 \\
\hline & $\mathbf{R F}$ & 9.04 & & $4.00-15.40$ & 5.86 & 6.75 & 75.41 & 3.15 \\
\hline \multirow[t]{2}{*}{ FZL } & IR & 84.67 & \multirow[t]{2}{*}{2.00} & $56.20-110.00$ & 7.71 & 9.21 & 70.19 & 12.25 \\
\hline & $\mathbf{R F}$ & 82.98 & & $7.20-118.75$ & 10.12 & 11.61 & 76.06 & 16.57 \\
\hline \multirow[t]{2}{*}{ MSL } & IR & 75.97 & \multirow[t]{2}{*}{4.24} & $5.00-106.80$ & 8.91 & 11.34 & 61.74 & 12.57 \\
\hline & $\mathbf{R F}$ & 72.75 & & $36.60-91.40$ & 7.56 & 8.68 & 75.81 & 11.56 \\
\hline \multirow[t]{2}{*}{ SMS } & IR & 51.19 & \multirow[t]{2}{*}{15.96} & $38.00-91.80$ & 10.82 & 11.45 & 89.28 & 15.07 \\
\hline & $\mathbf{R F}$ & 43.02 & & $29.40-65.20$ & 7.75 & 8.73 & 78.73 & 9.29 \\
\hline \multirow[t]{2}{*}{$\mathbf{S P}$} & IR & 319.01 & \multirow[t]{2}{*}{18.16} & $187.40-578.20$ & 31.10 & 36.42 & 72.89 & 97.68 \\
\hline & RF & 261.07 & & $127.40-485.80$ & 26.41 & 33.94 & 60.54 & 68.39 \\
\hline \multirow[t]{2}{*}{ SL } & IR & 4.50 & \multirow[t]{2}{*}{0.00} & $3.46-5.52$ & $*$ & $*$ & $*$ & * \\
\hline & $\mathbf{R F}$ & 4.50 & & $3.52-5.43$ & 1.25 & 1.48 & 71.43 & 0.46 \\
\hline \multirow[t]{2}{*}{ SPS } & IR & 16.39 & \multirow[t]{2}{*}{6.35} & $14.00-18.72$ & 2.09 & 2.32 & 81.15 & 1.57 \\
\hline & $\mathbf{R F}$ & 15.35 & & $13.70-18.10$ & 1.50 & 1.93 & 60.70 & 0.94 \\
\hline \multirow[t]{2}{*}{ BY } & IR & 95.23 & \multirow[t]{2}{*}{13.55} & $47.00-148.80$ & 18.16 & 21.85 & 69.04 & 30.33 \\
\hline & $\mathbf{R F}$ & 82.33 & & $40.32-161.60$ & 25.33 & 26.32 & 92.68 & 45.59 \\
\hline \multirow[t]{2}{*}{ SY } & IR & 29.72 & \multirow[t]{2}{*}{37.21} & $14.43-36.40$ & 8.56 & 9.48 & 81.62 & 8.69 \\
\hline & $\mathbf{R F}$ & 18.66 & & $7.19-26.50$ & 11.10 & 11.25 & 97.32 & 9.74 \\
\hline \multirow[t]{2}{*}{ TW } & IR & 6.11 & \multirow[t]{2}{*}{6.55} & $4.60-7.60$ & 2.49 & 2.71 & 84.41 & 1.17 \\
\hline & $\mathbf{R F}$ & 5.71 & & $3.68-8.16$ & 2.90 & 3.10 & 87.45 & 1.34 \\
\hline \multirow[t]{2}{*}{ Oil } & IR & 41.44 & \multirow[t]{2}{*}{0.56} & $38.24-43.70$ & 1.10 & 1.43 & 59.10 & 1.12 \\
\hline & RF & 41.21 & & $37.96-43.56$ & 1.52 & 1.61 & 88.94 & 1.89 \\
\hline
\end{tabular}

* Mean sum squares were non-significant for these characters; hence genetic parameters were not calculated

I=Irrigated, $\mathrm{RF}=$ Rainfed, $\mathrm{PH}=\mathrm{Plant}$ height, $\mathrm{PB}=$ Primary branches per plant, $\mathrm{SB}=$ Secondary branches per plant, FZL=Fruiting zone length, MSL=Main shoot length, SMS=Siliquae on main shoot, SP=Siliquae per plant, $\mathrm{SL}=$ Siliquae length, $\mathrm{SPS}=$ Seeds per siliqua, BY=Biological yield, $\mathrm{SY}=$ Seed yield per plant, $\mathrm{TW}=$ test weight

Table.2 Correlation coefficients between different characters in advanced progenies of Indian mustard under irrigated and rainfed conditions

\begin{tabular}{|c|c|c|c|c|c|c|c|c|c|c|c|c|c|c|}
\hline Trait & Condition & PH & PB & SB & FZL & MSL & SMS & SP & SL & SPS & BY & SY & TW & Oil \\
\hline \multirow[t]{2}{*}{ PH } & IR & 1.000 & & & & & & & & & & & & \\
\hline & RF & 1.000 & & & & & & & & & & & & \\
\hline \multirow[t]{2}{*}{ PB } & IR & 0.001 & 1.000 & & & & & & & & & & & \\
\hline & RF & $0.378^{* *}$ & 1.000 & & & & & & & & & & & \\
\hline \multirow[t]{2}{*}{ SB } & IR & -0.084 & $0.738^{* *}$ & 1.000 & & & & & & & & & & \\
\hline & $\mathbf{R F}$ & 0.319 ** & $0.563 * *$ & 1.000 & & & & & & & & & & \\
\hline \multirow[t]{2}{*}{ FZL } & IR & $0.312^{* *}$ & $0.316^{* *}$ & $0.285^{* *}$ & 1.000 & & & & & & & & & \\
\hline & RF & $0.503^{* *}$ & $0.254 * *$ & $0.324 * *$ & 1.000 & & & & & & & & & \\
\hline \multirow[t]{2}{*}{ MSL } & IR & $0.234^{* *}$ & $0.307^{* *}$ & $0.199 * *$ & $0.543 * *$ & 1.000 & & & & & & & & \\
\hline & $\mathbf{R F}$ & $0.535 * *$ & 0.092 & $0.279 * *$ & $0.515 * *$ & 1.000 & & & & & & & & \\
\hline \multirow[t]{2}{*}{ SMS } & IR & 0.009 & 0.075 & $0.178^{* * *}$ & $0.332 * *$ & $0.127^{*}$ & 1.000 & & & & & & & \\
\hline & RF & $0.382^{* *}$ & 0.101 & $0.186^{* *}$ & $0.412^{* * *}$ & $0.409^{* *}$ & 1.000 & & & & & & & \\
\hline \multirow[t]{2}{*}{ SP } & IR & -0.062 & $0.535 * *$ & $0.682^{* *}$ & 0.395 ** & 0.325 *** & $0.318^{* *}$ & 1.000 & & & & & & \\
\hline & $\mathbf{R F}$ & $0.291 * *$ & $0.357^{* *}$ & $0.533^{* *}$ & $0.414 * *$ & $0.270^{* *}$ & $0.415^{* *}$ & 1.000 & & & & & & \\
\hline \multirow[t]{2}{*}{ SL } & IR & -0.057 & 0.017 & -0.022 & -0.072 & $-0.119^{*}$ & 0.066 & -0.050 & 1.000 & & & & & \\
\hline & $\mathbf{R F}$ & -0.027 & -0.030 & 0.078 & -0.015 & -0.032 & -0.063 & 0.057 & 1.000 & & & & & \\
\hline \multirow[t]{2}{*}{ SPS } & IR & -0.048 & 0.027 & 0.017 & -0.013 & -0.041 & 0.060 & -0.066 & $0.610^{* *}$ & 1.000 & & & & \\
\hline & $\mathbf{R F}$ & $0.167^{*}$ & $0.205 * *$ & $0.318^{* *}$ & $0.124^{*}$ & $0.136^{*}$ & 0.112 & $0.288^{* * *}$ & 0.066 & 1.000 & & & & \\
\hline \multirow[t]{2}{*}{ BY } & IR & 0.023 & $0.420 * *$ & $0.466^{* * *}$ & $0.412 * *$ & $0.274 * *$ & $0.205^{* *}$ & $0.543 * *$ & 0.107 & 0.065 & 1.000 & & & \\
\hline & $\mathbf{R F}$ & $0.409^{* *}$ & $0.413^{* *}$ & $0.524^{* *}$ & $0.373 * *$ & $0.323^{* * *}$ & $0.382 * *$ & $0.583^{* *}$ & -0.068 & $0.291^{* * *}$ & 1.000 & & & \\
\hline \multirow[t]{2}{*}{ SY } & IR & -0.082 & $0.393 * *$ & $0.386^{* *}$ & $0.168^{* * *}$ & $0.131^{*}$ & $0.187^{* *}$ & $0.436^{* * *}$ & 0.098 & -0.012 & $0.600^{* * *}$ & 1.000 & & \\
\hline & $\mathbf{R F}$ & $0.432^{* *}$ & $0.433 * *$ & $0.406^{* * *}$ & $0.405^{* * *}$ & $0.426^{* * *}$ & $0.430^{* *}$ & $0.554 * *$ & $-0.127 *$ & 0.201 ** & $0.712^{* *}$ & 1.000 & & \\
\hline \multirow[t]{2}{*}{$\mathrm{TW}$} & IR & 0.018 & 0.004 & -0.073 & $-0.167^{* *}$ & 0.026 & -0.040 & -0.058 & 0.065 & -0.087 & -0.021 & 0.095 & 1.000 & \\
\hline & $\mathbf{R F}$ & $0.213^{* *}$ & $0.180^{* *}$ & $0.151^{* *}$ & $0.146^{*}$ & $0.260^{* * *}$ & $0.349^{* *}$ & $0.168^{* * *}$ & -0.087 & -0.002 & $0.293^{* *}$ & $0.353^{* *}$ & 1.000 & \\
\hline \multirow[t]{2}{*}{ Oil } & IR & 0.036 & -0.022 & -0.054 & $-0.140 *$ & 0.009 & 0.028 & $-0.146^{*}$ & $0.238^{* *}$ & $0.433^{* * *}$ & 0.036 & 0.074 & $-0.166^{* *}$ & 1.000 \\
\hline & $\mathbf{R F}$ & $-0.132 *$ & $-0.144 *$ & $-0.208 * *$ & $-0.119 *$ & $-0.222 * *$ & $-0.177^{*}$ & $-0.209^{* *}$ & $0.291 * *$ & -0.019 & $-0.257 * *$ & $-0.248^{* *}$ & $-0.242 * *$ & 1.000 \\
\hline
\end{tabular}

$\mathrm{I}=$ Irrigated, $\mathrm{RF}=$ Rainfed, $\mathrm{PH}=$ Plant height, $\mathrm{PB}=$ Primary branches per plant, $\mathrm{SB}=$ Secondary branches per plant, $\mathrm{FZL}=$ Fruiting zone length, MSL=Main shoot length, $\mathrm{SMS}=$ Siliquae on main shoot, $\mathrm{SP}=$ Siliquae per plant, $\mathrm{SL}=$ Siliquae length, SPS=Seeds per siliqua, BY=Biological yield, $\mathrm{SY}=$ Seed yield per plant, $\mathrm{TW}=$ test weight and oil content 
Int.J.Curr.Microbiol.App.Sci (2018) 7(3): 2519-2526

Table.3 Seed yield (g/plant), DSI values and relative yield of Indian mustard genotypes under irrigated and rainfed conditions

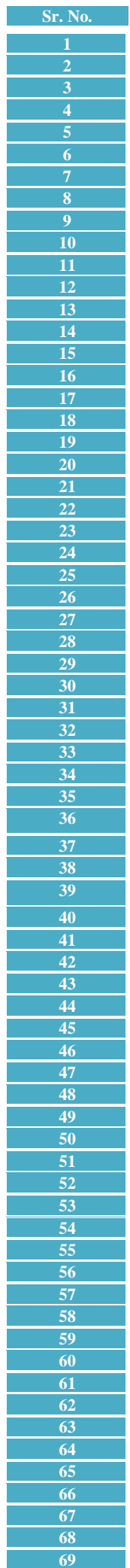

\begin{tabular}{|c|c|c|c|c|c|}
\hline Genotypes & SY (IR) & SY (RF) & DSI & RY (IR) & RY (RF) \\
\hline BPR-1716-9-7 & 30.35 & 25.20 & 0.456 & 0.83 & 0.95 \\
\hline BPR-1717-11-9 & 25.35 & 14.61 & 1.138 & 0.70 & 0.55 \\
\hline BPR-1717-14-12 & 32.91 & 21.18 & 0.958 & 0.90 & 0.80 \\
\hline BPR-1721-28-23 & 20.75 & 22.17 & -0.184 & 0.57 & 0.84 \\
\hline BPR-1721-33-28 & 26.22 & 23.15 & 0.315 & 0.72 & 0.87 \\
\hline BPR-1722-53-40 & 30.37 & 24.60 & 0.511 & 0.83 & 0.93 \\
\hline BPR-1724-66-47 & 28.19 & 26.32 & 0.178 & 0.77 & 0.99 \\
\hline BPR-1724-72-51 & 27.40 & 22.33 & 0.497 & 0.75 & 0.84 \\
\hline BPR-1723-58-60 & 18.41 & 21.06 & -0.386 & 0.51 & 0.79 \\
\hline BPR-1566-4-63 & 22.30 & 24.96 & -0.321 & 0.61 & 0.94 \\
\hline BPR-1480-12-66 & 35.60 & 15.95 & 1.483 & 0.98 & 0.60 \\
\hline BPR-1686-29-76 & 36.09 & 20.43 & 1.166 & 0.99 & 0.77 \\
\hline DHS-13-102 & 34.38 & 22.76 & 0.908 & 0.94 & 0.86 \\
\hline DHS-14-103 & 24.42 & 26.50 & -0.229 & 0.67 & 1.00 \\
\hline DHS-18-104 & 22.93 & 25.14 & -0.259 & 0.63 & 0.95 \\
\hline BPR-1722-52-39 & 22.11 & 12.37 & 1.184 & 0.61 & 0.47 \\
\hline BPR-1722-42-58 & 28.87 & 20.32 & 0.796 & 0.79 & 0.77 \\
\hline BPR-1686-34-80 & 34.21 & 21.30 & 1.014 & 0.94 & 0.80 \\
\hline BPR-1686-39-83 & 25.81 & 25.94 & -0.014 & 0.71 & 0.98 \\
\hline BPR-1684-42-84 & 32.40 & 25.45 & 0.576 & 0.89 & 0.96 \\
\hline BPR-1688-54-91 & 31.94 & 17.88 & 1.183 & 0.88 & 0.67 \\
\hline BPR-1688-55-92 & 32.65 & 19.66 & 1.069 & 0.90 & 0.74 \\
\hline BPR-1684-58-94 & 33.02 & 16.46 & 1.347 & 0.91 & 0.62 \\
\hline BPR-1616-61-95 & 14.43 & 13.20 & 0.229 & 0.40 & 0.50 \\
\hline BPR-1686-68-97 & 33.32 & 10.91 & 1.807 & 0.92 & 0.41 \\
\hline BPR-1676-63-96 & 33.01 & 23.87 & 0.744 & 0.91 & 0.90 \\
\hline BPR-1566-7-101 & 16.59 & 23.11 & -1.056 & 0.46 & 0.87 \\
\hline DHS-23-105 & 31.36 & 22.50 & 0.759 & 0.86 & 0.85 \\
\hline DHS-24-107 & 32.15 & 19.93 & 1.021 & 0.88 & 0.75 \\
\hline DHS-28-108 & 35.60 & 17.98 & 1.330 & 0.98 & 0.68 \\
\hline DHS-29-109 & 36.40 & 19.21 & 1.269 & 1.00 & 0.72 \\
\hline DFS-1-110 & 29.32 & 22.32 & 0.642 & 0.81 & 0.84 \\
\hline DFS-2-111 & 33.88 & 26.45 & 0.589 & 0.93 & 1.00 \\
\hline DFS-9-112 & 32.38 & 18.05 & 1.189 & 0.89 & 0.68 \\
\hline DFS-11-113 & 35.25 & 25.65 & 0.732 & 0.97 & 0.97 \\
\hline DFS-18-114 & 31.25 & 23.55 & 0.662 & 0.86 & 0.89 \\
\hline DFS-21-115 & 17.54 & 21.54 & -0.613 & 0.48 & 0.81 \\
\hline DFS-22-116 & 22.21 & 17.27 & 0.598 & 0.61 & 0.65 \\
\hline YHS-26-117 & 28.81 & 14.81 & 1.306 & 0.79 & 0.56 \\
\hline YHS-36-119 & 31.06 & 12.88 & 1.573 & 0.85 & 0.49 \\
\hline YHS-43-120 & 30.90 & 26.48 & 0.385 & 0.85 & 1.00 \\
\hline YHS-44-121 & 20.78 & 22.50 & -0.222 & 0.57 & 0.85 \\
\hline YHS-57-122 & 34.85 & 20.00 & 1.145 & 0.96 & 0.75 \\
\hline YHS-113-123 & 33.35 & 19.06 & 1.151 & 0.92 & 0.72 \\
\hline BPR-1741-2-1 & 35.44 & 20.93 & 1.100 & 0.97 & 0.79 \\
\hline BPR-1541-3-2 & 32.14 & 11.16 & 1.754 & 0.88 & 0.42 \\
\hline BPR-1741-4-2 & 31.60 & 17.27 & 1.218 & 0.87 & 0.65 \\
\hline BPR-1741-5-1 & 35.22 & 24.96 & 0.783 & 0.97 & 0.94 \\
\hline BPR-1741-6-1 & 35.21 & 24.22 & 0.839 & 0.97 & 0.91 \\
\hline BPR-1741-7-2 & 21.25 & 18.73 & 0.319 & 0.58 & 0.71 \\
\hline BPR-1741-8-1 & 34.30 & 14.14 & 1.580 & 0.94 & 0.53 \\
\hline BPR-1741-9-1 & 29.38 & 10.84 & 1.696 & 0.81 & 0.41 \\
\hline BPR-1741-9-2 & 35.29 & 7.19 & 2.140 & 0.97 & 0.27 \\
\hline BPR-1741-10-2 & 28.93 & 23.44 & 0.510 & 0.79 & 0.88 \\
\hline BPR-1741-11-1 & 33.76 & 15.45 & 1.457 & 0.93 & 0.58 \\
\hline BPR-1741-12-1 & 30.18 & 20.04 & 0.903 & 0.83 & 0.76 \\
\hline BPR-1741-12-3 & 33.20 & 22.00 & 0.907 & 0.91 & 0.83 \\
\hline BPR-1741-13-2 & 24.81 & 18.63 & 0.670 & 0.68 & 0.70 \\
\hline BPR-1741-14-2 & 29.89 & 22.67 & 0.649 & 0.82 & 0.86 \\
\hline BPR-1741-17-1 & 32.73 & 25.52 & 0.592 & 0.90 & 0.96 \\
\hline BPR-1741-18-1 & 35.24 & 24.50 & 0.819 & 0.97 & 0.92 \\
\hline BPR-1741-19-1 & 23.58 & 17.05 & 0.744 & 0.65 & 0.64 \\
\hline BPR-1741-19-2 & 34.19 & 22.84 & 0.892 & 0.94 & 0.86 \\
\hline BPR-1741-20-1 & 32.50 & 13.15 & 1.600 & 0.89 & 0.50 \\
\hline BPR-1741-21-1 & 33.75 & 14.33 & 1.546 & 0.93 & 0.54 \\
\hline BPR-1741-21-1 & 31.25 & 12.05 & 1.651 & 0.86 & 0.45 \\
\hline BPR-1741-24-4 & 33.65 & 13.55 & 1.605 & 0.92 & 0.51 \\
\hline BPR-1741-25-1 & 34.85 & 9.16 & 1.981 & 0.96 & 0.35 \\
\hline BPR-1741-26-5 & 29.22 & 21.65 & 0.696 & 0.80 & 0.82 \\
\hline
\end{tabular}




\begin{tabular}{|c|c|c|c|c|c|c|}
\hline 70 & BPR-1741-27-2 & 33.62 & 20.32 & 1.063 & 0.92 & 0.77 \\
\hline 71 & BPR-1741-29-1 & 30.25 & 20.65 & 0.853 & 0.83 & 0.78 \\
\hline 72 & BPR-1741-30-3 & 23.37 & 16.03 & 0.844 & 0.64 & 0.60 \\
\hline 73 & BPR-1741-30-4 & 22.78 & 22.03 & 0.088 & 0.63 & 0.83 \\
\hline 74 & BPR-1741-31-1 & 29.52 & 14.98 & 1.324 & 0.81 & 0.57 \\
\hline 75 & BPR-1741-32-1 & 34.41 & 11.95 & 1.754 & 0.95 & 0.45 \\
\hline 76 & BPR-1741-32-3 & 32.66 & 15.23 & 1.434 & 0.90 & 0.57 \\
\hline 77 & BPR-1741-33-2 & 27.64 & 13.84 & 1.342 & 0.76 & 0.52 \\
\hline 78 & BPR-1741-34-2 & 21.22 & 19.23 & 0.252 & 0.58 & 0.73 \\
\hline 79 & BPR-1741-35-2 & 28.68 & 12.54 & 1.512 & 0.79 & 0.47 \\
\hline 80 & BPR-1741-36-1 & 26.70 & 13.24 & 1.355 & 0.73 & 0.50 \\
\hline 81 & BPR-1741-40-2 & 34.10 & 19.48 & 1.152 & 0.94 & 0.74 \\
\hline 82 & BPR-1741-40-1 & 19.55 & 11.71 & 1.078 & 0.54 & 0.44 \\
\hline 83 & BPR-1741-44-2 & 20.14 & 22.49 & -0.314 & 0.55 & 0.85 \\
\hline 84 & BPR-1741-85-1 & 28.71 & 10.08 & 1.744 & 0.79 & 0.38 \\
\hline 85 & BPR-1741-85-2 & 29.36 & 17.85 & 1.053 & 0.81 & 0.67 \\
\hline 86 & BPR-174188-2 & 30.25 & 11.52 & 1.664 & 0.83 & 0.43 \\
\hline 87 & BPR-1741-90-1 & 29.09 & 11.99 & 1.580 & 0.80 & 0.45 \\
\hline 88 & BPR-1741-90-2 & 34.25 & 11.15 & 1.812 & 0.94 & 0.42 \\
\hline 89 & BPR-1741-91-1 & 35.21 & 21.54 & 1.043 & 0.97 & 0.81 \\
\hline 90 & BPR-1741-91-2 & 31.61 & 20.84 & 0.916 & 0.87 & 0.79 \\
\hline 91 & BPR-1741-94-1 & 30.84 & 21.31 & 0.831 & 0.85 & 0.80 \\
\hline 92 & BPR-1741-96-2 & 28.71 & 20.54 & 0.765 & 0.79 & 0.78 \\
\hline 93 & BPR-1741-97-2 & 16.87 & 22.85 & -0.953 & 0.46 & 0.86 \\
\hline 94 & BPR-1741-98-2 & 22.32 & 13.02 & 1.119 & 0.61 & 0.49 \\
\hline 95 & BPR-1741-101-1 & 27.90 & 20.73 & 0.690 & 0.77 & 0.78 \\
\hline 96 & BPR-1741-102-1 & 31.84 & 12.32 & 1.647 & 0.87 & 0.46 \\
\hline 97 & BPR-1741-102-2 & 29.50 & 17.65 & 1.079 & 0.81 & 0.67 \\
\hline 98 & BPR-1741-104-1 & 33.62 & 9.30 & 1.944 & 0.92 & 0.35 \\
\hline 99 & BPR-1741-108-1 & 30.25 & 20.44 & 0.872 & 0.83 & 0.77 \\
\hline \multirow[t]{3}{*}{100} & BPR-1741-109-2 & 23.06 & 14.18 & 1.035 & 0.63 & 0.54 \\
\hline & Mean & 29.41 & 18.74 & 0.90 & 0.81 & 0.71 \\
\hline & Range & $14.43-36.40$ & $7.19-26.50$ & $-1.06-2.14$ & $0.40-1.00$ & $0.27-1.00$ \\
\hline
\end{tabular}

SY=seed yield, DSI=drought susceptibility index, RY=relative yields.

Therefore, for efficient use of scarce water resource high yielding genotypes can be developed with long siliqua length, more siliqua on main shoot, more seeds siliqua ${ }^{-1}$, test weight and fast-growing seedling traits. Under irrigated conditions, seed yield plant ${ }^{-1}$ was negative but significantly correlated with plant height ($0.082)$, seeds siliqua ${ }^{-1}(-0.012)$ and oil content (0.092). Association between some characters was non-significant which implies that the two variables are not linearly related on these two may be related but in a non-linear fashion (Gomez and Gomez, 1980).

Drought susceptibility index (DSI) was calculated for each genotype as a criterion of drought tolerance. The lowest value indicates highest level of drought tolerance and viceversa. The DSI ranged from -1.056 (BPR-15667-101) to 2.140 (BPR-1741-9-2) (Table 3). The genotypes BPR-1566-7-101, BPR-1741-97-2, DFS-21-115, BPR-1723-58-60, BPR-1566-463, BPR-1741-44-2, DHS-18-104, DHS-14103, YHS-44-121, BPR-1721-28-23 and BPR1686-39-83 had lower DSI values $(<$ or $\sim 0.00)$, thus can be as drought tolerant. A total 40 genotypes were found moderately drought tolerant (>0.0-1.0), 30genotypes ranked as moderately susceptible (>1.0-1.5) and 19 genotypes as susceptible (>1.5) (Table 3). Clarke et al., (1984) opined that selection for yield under dry condition should alone be more productive avenue for improvement of drought resistance until more rapid and effective screening procedures could be developed.

As DSI is a ratio, a genotype could have lower value of this index even when its mean seed yield under drought condition is significantly low than better performing genotype (s). Therefore, genotypes in present study were selected on the basis of high mean seed yield along with lower DSI value. DSI values and seed yield under drought conditions as a selection criterion in Indian mustard has been earlier used by Singh and Choudhary (2003) and Chauhan et al., (2007).

Mean relative yield under irrigated condition is more than rain fed-condition (Table 3 ). Top ten 
genotypes viz., DHS-29-109, BPR-1686-29-76, BPR-1480-12-66, DHS-28-108, DFS-11-113, BPR-1741-2-1, BPR-1741-5-1, BPR-1741-6-1, BPR-1741-9-2 and BPR-1741-18-1 were relatively higher yielding under irrigated $(\mathrm{RY}>$ mean RY). The top ten genotypes DHS-14-103, DFS-2-111, YHS-43-120, BPR-1724-66-47, DHS-18-104, BPR-1617-9-7, BPR-1686-39-83, DFS-11-113, BPR-1684-42-84 and BPR-15664-63 were relatively high yielding under rainfed conditions. Relative yield may be used to evaluate the yield potential of genotypes under water stress conditions.

Higher relative yield indicate that genotypes performed relatively well under drought. The higher mean RY under drought showed the adaptiveness of genotypes to moisture stress.

The genotypes which showed higher RY under stress indicates their adaptive nature.

It is actually a relative mean where single inferior genotype might affect the overall mean and therefore it requires confirmation by evaluating under varying moisture stress condition.

Genotypes showed higher RY under irrigated condition and they indicated their susceptibility to drought. The results indicated that there is urgent need to breed genotypes of mustard separately for varied conditions.

\section{References}

Abhishek, R., R. Prasad and Gupta, V. K. 2004. Computer aided construction and analysis of augmented designs. Journal Indian Society Agriculture Statistics. 57: 320344.

Ahmed, R., J. C. Stark, A. Tanveer and Mustafa, T. 1999. Yield potential and stability indices to evaluate spring wheat genotypes under drought. Agricultural Sciences. 4: 53-59.

Burton, G. W. 1952. Quantitative inheritance of grasses. Proceedings of 6th International Grassland Congress. 1: 227-283.
Chauhan, J. S., M. K. Tyagi, A. Kumar, N. I. Nashaat, M. Singh, N. B. Singh, M. L. Jakhar and Welham, S. J. 2007. Drought effects on yield and its components in Indian mustard (Brassica juncea L.). Plant Breeding. 126: 399-402.

Clarke, J., Townley-Smith, T. F., McCaaig, T. N. and Green, D. M. 1984. Growth analysis of spring wheat cultivars of varying drought resistance. Crop Science. 56: 603-626.

Doddabhimappa, R., B. Gangapur, G. Prakash, G. P. Salimath, R. L. Ravikumar and Rao, M. S. L. 2009. Correlation and path analysis in Indian mustard (Brassica juncea L. Czern and Coss). Plant Breeding. 22 (5): 971-977.

Fedrer, W. T. 1956. Augmented Design Karnataka Journal of Agriculture Science. Hawain Planters Record. 20: 191-207.

Fischer, R. A., and Maurer, R. 1978. Drought resistance in spring wheat cultivars. 1. Grain yield responses. Australian Journal of Agricultural Research. 29: 897-912.

Gomez, K. A., and Gomez, A. A. 1980. Statistical Procedures for Agriculture Research. John Wiley and Sons Inc., New York.

Johnson, H. W., H. F. Robinson and Comstock, R. E. 1955. Estimate of genetic and environmental variability in soybean. Agronomy Journal. 47: 314-318.

Kardam, D. K., and Singh, V. V. 2005. Correlation and path coefficient analysis in Indian mustard (Brassica juncea L.) grown under rainfed condition. Journal Spice Aromatics Crops. 14: 56-60.

Kumawat, B. L., D. D. Sharma and Jat, S. C. 1997. Effect of Brassinosteroid on yield and yield attributing characteristics under water deficit stress condition in mustard (B. juncea L.). Annals of Biology. 13: 9193.

Mamun-Hossain, A. R. M. and, Joarder, N. 1987. Studies on some mechanical cells in the basal internode of some rice cultivars in relation to lodging. Pakistan Journal Agriculture Research. 8: 24-28. 
Meena, H. S, A. Kumar, V. V. Singh, P. D. Meena, B. Ram and Kulshrestha, S. 2017. Genetic variability and inter-relation of seed yield with contributing traits in Indian mustard (Brassica juncea). Journal of Oilseed Brassica. 8 (2): 131-137.

Meena, H. S., B. Ram, A. Kumar, B. K. Singh, P. D. Meena, V. V. Singh, and Singh, D. 2014. Heterobeltiosis and standard heterosis for seed yield and important traits in Brassica juncea. Journal of Oilseed Brassica. 5:134-140.

Meena, S. S., R. Yadav and Singh, V. V. 2008. Genetic variability for seed and seedling traits in the advance breeding lines of Indian mustard [Brassica juncea (L.) Czern. \& Coss.]. Seed Research. 36: 152156.

Patel, J. M., K. M. Patel, C. J. Patel and Prajapati, K. P. 2006. Genetic parameter and inter relationship analysis in Indian mustard (Brassica juncea L.). Journal of Oilseed Research. 23(2): 159-160.

Ramya, B., K. Saravanan and Kumar, P. S. 2012. Genetic parameters of variability, correlation and path coefficient studies for grain yield and other yield attribute traits in rice (Oryza sativa L.). Plant Archieves. 12: 711-715.

Resketo, P., and Szabo, L. 1992. The effect of drought on development and yield components of soybean. International Journal of Tropical Agriculture. 8: 34754.

Richards, R. A. 1978. Genetic analysis of drought stress response in rapeseed $(B$. campestris and B. napus). Eupytica. 27: 609-615.

Roy, R. K., A. Kumar, S. Kumar and Kumar, A. 2016. Genetic variability, correlation, path analysis and genetic diversity studies in late sown mustard (Brassica juncea $\mathrm{L}$. Czern \& Coss.). Plant Breeding. 4: 35-45.

Singh, K. H., R. K. Mahawar and Kumar A. 2007. Relationship between floral and agronomic traits in Indian mustard (Brassica juncea). Wuhan (China). Sci. Press. USA Inc: 53.

Singh, S. P., and Choudhary, A. K. 2003. Selection criteria for drought tolerance in Indian mustard (Brassica juncea L.). Indian Journal of Genetics and Plant Breeding. 63: 263-264.

Singh, V. V., M. L. Meena, B. R. Singh and Singh, D. 2015. Patterns of Genetic Variation, Correlations under Irrigated and Rainfed Conditions in Indian mustard [Brassica juncea (L.) Czern. \& Coss.]. Indian Journal Plant Genetics Resources. 28 (2): 198-204.

Singh, V. V., P. K. Rai, S. A. Siddiqui, V. Verma and Yadav, R. 2011. Genetic variability and relative drought tolerance in interspecific progenies of Brassica juncea. Agriculture and Biology Journal of North America. 2 (1):34-41.

Singh, V. V., S. Singh, V. Verma, S. S. Meena and Kumar, A. 2009. Genetic variability for seedling traits in Indian mustard under moisture stress conditions. Indian Journal of Plant Genetics Resources. 22 (1): 4649.

Tiwari, A. K., S. K. Singh, A. Tomar and Singh, M. 2017. Heritability, genetic advance and correlation coefficient analysis in Indian mustard (Brassica Juncea (L.) Czern \& Coss). Journal of Pharmacognosy and Phytochemistry. 6 (1): 356-359.

\section{How to cite this article:}

Singh, V.V., Pankaj Garg, H.S. Meena and Meena, M.L. 2018. Drought Stress Response of Indian Mustard (Brassica juncea L.) Genotypes. Int.J.Curr.Microbiol.App.Sci. 7(03): 2519-2526. doi: https://doi.org/10.20546/ijcmas.2018.703.291 